\title{
Questes
}

\section{L'Apocalypse d'Isabelle de France (1313) et son lien avec un groupe de Bibles historiales}

\section{Louis-Patrick Bergot}

\section{(2) OpenEdition}

Journals

\section{Édition électronique}

URL : http://journals.openedition.org/questes/4860

DOI : 10.4000/questes.4860

ISSN : 2109-9472

\section{Éditeur}

Les Amis de Questes

\section{Édition imprimée}

Date de publication : 1 juillet 2018

Pagination : 63-79

ISSN : 2102-7188

\section{Référence électronique}

Louis-Patrick Bergot, «L'Apocalypse d'Isabelle de France (1313) et son lien avec un groupe de Bibles

historiales », Questes [En ligne], 38 | 2018, mis en ligne le 18 juin 2018, consulté le 01 mai 2019. URL: http://journals.openedition.org/questes/4860 ; DOI : 10.4000/questes.4860 


\section{L'Apocalypse d'Isabelle de France (1313) et son lien avec un groupe de Bibles historiales}

\section{Louis-Patrick BERGOT}

Université Paris-Sorbonne

La transmission textuelle de l'Apocalypse en français au Moyen Âge est d'une telle complexité qu'il conviendrait de la représenter comme une mosaïque constituée de plusieurs centaines de pièces, dont l'ampleur serait d'autant plus difficile à saisir que la plupart de ces pièces sont aujourd'hui perdues.

Paradoxalement, il n'existe que très peu de travaux critiques sur la transmission textuelle de l'Apocalypse au Moyen Âge, alors que les études qui traitent de sa réception iconographique se comptent par dizaines ${ }^{1}$. En ce qui concerne la tradition manuscrite, on n'en sait guère plus qu'en 1901, au moment où paraissait un ouvrage fondateur sur le sujet, dû au travail de Léopold Delisle et de Paul Meyer, intitulé L'Apocalypse en français au XIII siècle ${ }^{2}$. Il s'agit de l'édition, par Paul Meyer, d'une version glosée (nous l'appellerons G), qui était très populaire au Moyen Âge, puisqu'on en possède une quarantaine de

\footnotetext{
${ }^{1}$ Sur l'iconographie médiévale de l'Apocalypse, on consultera les travaux, trop nombreux pour être cités en détail, de George Henderson, de Peter Klein, d'Yves Christe, de Nigel Morgan, de Suzanne Lewis, et plus récemment de Natasha O'Hear et de Renana Bartal.

${ }^{2}$ Léopold Delisle et Paul Meyer, L’Apocalypse en français au XIII siècle, Paris, Didot, 1901.
} 
copies. L'un des témoins les plus célèbres de cette version est le manuscrit fr. 403 de la BnF, sur lequel s'appuie l'édition de Paul Meyer³ .

Dans l'introduction à son édition, Paul Meyer distingue également quatre versions non glosées, auxquelles il donne les lettres $\mathrm{A}, \mathrm{B}, \mathrm{C}$ et $\mathrm{D}^{4}$. Dans le cadre de nos recherches, nous avons décidé d'étendre ce classement à l'ensemble des traductions françaises de l'Apocalypse au Moyen Âge. Entre le XIII ${ }^{\mathrm{e}}$ et le $\mathrm{XV}^{\mathrm{e}}$ siècle, il existe une quinzaine de traductions différentes, chacune d'entre elles étant le fruit d'une initiative individuelle ou collective qui lui est propre. Nous leur avons attribué des lettres, de A à W. Quelques-unes de ces traductions sont versifiées, notamment les versions $\mathrm{K}, \mathrm{M}, \mathrm{R}$ et $\mathrm{W}^{5}$; toutes les autres sont en prose et, parmi elles, il faut distinguer celles qui sont pourvues d'une glose ${ }^{6}(\mathrm{H}, \mathrm{I}, \mathrm{J}$

\footnotetext{
${ }^{3}$ Dans son édition, Paul Meyer donne également les variantes des manuscrits Paris, BnF, fr. 9574 et Arsenal, ms. 5214. Pour plus de précisions sur la version G, on consultera avec intérêt l'article de Daron Burrows, "Vers une nouvelle édition de l'Apocalypse en prose », dans Anglo-français. Philologie et linguistique, dir. Oreste Floquet et Gabriele Giannini, Paris, Classiques Garnier, 2015, p. 9-34. On trouvera dans cet article un inventaire de 40 témoins. Cette liste est encore incomplète. Il faudrait y ajouter deux manuscrits tardifs, des années 1470-1480: Glasgow, University of Glasgow, Hunterian Library, ms. 398 (V.2.18) (olim P.2.13), et New York, Pierpont Morgan Library, M.68.

${ }^{4}$ Voir Léopold Delisle et Paul Meyer, L’Apocalypse en français au XIII siècle, op. cit., p. CCXXX-CCXLIX. Il ne faut pas confondre les versions A, B, C et D avec les manuscrits A (fr. 403), B (fr. 9574) et C (Arsenal, ms. 5214), qui sont trois manuscrits de la version $\mathrm{G}$.

5 - K (1 ms.) : Henry A. Todd, «The Old French Versified Apocalypse of the Kerr Manuscript », Publications of the Modern Language Association of America, vol. 18, $\mathrm{n}^{\mathrm{o}} 4,1903$, p. 535-577 ;

- M (1 ms.) : La Bible de Macé de La Charité, vol. 7, Apocalypse, dir. Jean Robert Smeets, éd. Reinier L. H. Lops, Leiden, Brill, 1982 ;

- R (10 mss.) : Revelacion (BL Royal 2.D.xiii), an Anglo-Norman Apocalypse Poem, éd. Brent A. Pitts, London, Anglo-Norman Text Society, 2010 ;

- W (1 ms.) : An Anglo-Norman Rhymed Apocalypse with Commentary, éd. Olwen Rhys, Oxford, Blackwell, 1946.

Sur les versions rimées, on pourra consulter l'article de Brent Pitts, «Versions of the Apocalypse in Medieval French Verse », Speculum, vol. 58, n 1, janvier 1983, p. 3159.

${ }^{6}$ Quand nous parlons de glose, il ne s'agit ni d'une glose marginale ni d'une glose interlinéaire (une pratique courante au XII ${ }^{\mathrm{e}}$ siècle). L'exégèse des versions " glosées »
} 
et $\mathrm{S}^{7}$ ) et celles qui au contraire ne sont pas glosées (A, B, C, D, E et $\mathrm{F}^{8}$ ). Ainsi, les années 1220-1350 constituent un âge d'or pour le texte de l'Apocalypse : durant cette période, les médiévaux aiment le traduire et le retraduire.

Dans le travail qui suit, nous souhaiterions nous pencher sur la version $\mathrm{A}^{9}$. Elle se trouve dans sept manuscrits :

- d'une part, dans un manuscrit conservé à la BnF (fr. 13096), qui ne comporte que l'Apocalypse et qui fut probablement réalisé en 1313 pour Isabelle de France, fille de Philippe IV et reine d'Angleterre de 1308 à 1327 ;

- d'autre part, à la fin de six Bibles historiales produites dans les années 1350-1380, notamment sous le règne de Charles V.

Ce que nous voudrions élucider, c'est le lien qui unit l'Apocalypse $^{10}$ d'Isabelle de France et cet ensemble de six Bibles historiales. Le rapport qu'entretiennent ces deux corpus suppose d'engager une réflexion sur la question des formes et des usages de la Bible au Moyen Âge. L'Apocalypse d'Isabelle et les Bibles historiales

fonctionne par groupes de versets (Apocalypse, I, 1-8 ; 9-16; 17-20 par exemple), chaque bloc étant systématiquement suivi d'une glose correspondante.

7 - H (1 ms.) : Apocalypse du Trinity College de Cambridge (R.16.2) ;

- I (3 mss.) : Paris, BnF, fr. 1768 ; Chantilly, Musée Condé, ms. 28 ; New York, Pierpont Morgan Library, M.133 (voir Léopold Delisle et Paul Meyer, L'Apocalypse en français au XIII siècle, op. cit., p. CCLIII-CCLVI) ;

- J (1 ms.) : Apocalypse d'Abingdon (Londres, BL, Add. 42555) ;

- S (1 ms.) : Bible de Jehan Servion (Lausanne, BCU, U 986, p. 476-555).

${ }^{8}$ - A $(7$ mss.) : voir infra ;

- B (3 mss.) : Paris, BnF, fr. 1036 ; fr. 24728 ; Vatican, BAV, Pal. lat. 1957 ;

- C (63 mss.) : cette version populaire se rencontre presque exclusivement dans les Bibles du XIII siècle et les Bibles historiales ;

- D (1 ms.) : Londres, BL, Add. 17333 ;

- E (1 ms.) : Paris, BnF, fr. 907, fol. 105vb-120rb ;

- F (1 ms.) : Philadelphia, Free Library, Widener 2, fol. 374va-383vb.

${ }^{9}$ Sur cette version, voir Léopold Delisle et Paul Meyer, L'Apocalypse en français au XIII siècle, op. cit., p. CCXXX-CCXXXVII.

${ }^{10}$ Nous mettons «Apocalypse » en caractères italiques quand il s'agit du manuscrit fr. 13096 de la BnF. 
divergent quant à la forme choisie: dans le manuscrit fr. 13096, l'Apocalypse est isolée, commentée et accompagnée d'un riche programme iconographique; dans les Bibles historiales, l'Apocalypse n'est généralement pas glosée et bénéficie d'une iconographie plus modeste. Mais l'Apocalypse d'Isabelle et les Bibles historiales se différencient également quant à leur usage : séparées dans le temps par plusieurs décennies, l'Apocalypse de 1313 et les six Bibles historiales que nous aborderons ne furent pas produites pour les mêmes commanditaires. En l'occurrence, nous verrons que ces deux corpus ont une portée politique qui rend compte des logiques d'alliance et de rivalité qui existaient entre les royaumes de France et d'Angleterre au début et au milieu du XIV ${ }^{\mathrm{e}}$ siècle.

Après une présentation des différents manuscrits, nous effectuerons une comparaison formelle entre les deux textes, celui d'Isabelle et celui de Charles V, afin de comprendre comment, mais surtout pourquoi - autrement dit pour quel usage -, la version A s'est retrouvée dans un groupe de six Bibles historiales, alors que ce n'était pas la traduction que l'on rencontrait habituellement dans ce type d'ouvrages.

\section{Présentation du manuscrit fr. 13096 de la BnF}

Pour bien saisir la forme et l'usage de l'Apocalypse d'Isabelle de France, il convient premièrement de présenter la structure et le contenu du manuscrit Paris, BnF, fr. $13096^{11}$. Il s'agit d'un volume de

\footnotetext{
${ }^{11}$ Il existe un fac-similé de ce manuscrit: Apocalipsis 1313, Madrid, M. Moleiro, 2006. Ce fac-similé est accompagné d'un volume de commentaires, où l'on trouvera des informations d'ordre codicologique et contextuel. Le manuscrit est également consultable sur le site Gallica :

http://gallica.bnf.fr/ark:/12148/btv1b10533304x.r=13096.
} 
167 feuillets, produit en 1313, comme l'indique le colophon ${ }^{12}$. Au fol. 1v se trouve un frontispice, qui représente saint Jean l'Évangéliste, plongé dans une cuve d'huile bouillante sur l'ordre de l'empereur Domitien. Sur la page de droite (fol. 2r), un prologue relate cet épisode : «Johans, por le preechement del evangelie, fut tenus de Domicien et devant la Porte Latine mis en un tonel de builhant oile» (fol. 2ra). Le texte de l'Apocalypse proprement dite ne débute qu'à la page suivante: «L'Apocalypsis Jhesu Christi, cui Dieus li donat por aovrir a ses serfs les choses cui tost covient estre. Et ilh le saielat; si l'envoiat parmi son angle $\gg($ fol. $2 \mathrm{vb})$.

Du début à la fin de l'Apocalypse, le texte biblique est placé sur la page de gauche (verso), tandis que la page de droite (recto) est occupée par une enluminure en pleine page illustrant le texte qui lui fait face. Parfois, une miniature est ajoutée dans la partie inférieure de la page de gauche. Ces miniatures supplémentaires, auxquelles n'est réservé qu'un tiers de la page, nous montrent l'Évangéliste dans diverses postures, en train de se ronger les ongles, de tailler sa plume ou de se concentrer pour recevoir la vision spirituelle de l'Apocalypse ${ }^{13}$. Ces miniatures servent de lien entre le texte (page de gauche) et l'enluminure (page de droite) : elles permettent d'introduire le regard du lecteur à la contemplation des visions apocalyptiques.

L'Apocalypse se termine au fol. 85v. Sur la page de droite (fol. 86r), nous pouvons de nouveau admirer un frontispice, qui représente les pécheurs contraints d'aller vers une gueule d'enfer. Ce frontispice est suivi d'une double page (fol. 86v-87r), où les damnés sont

\footnotetext{
12 «L'an del Incarnation .m. ccc. \& xiij. le samedi apres le sain Donis [sic] fut parfais cis Apokapse [sic]. Colins Chadewe l'ordinat et l'enluminat» (Paris, BnF, fr. 13096, fol. 167r).

${ }^{13}$ Pour plus de précisions, voir l'article que Suzanne Lewis a consacré à l'Apocalypse d'Isabelle de France : Suzanne Lewis, «The Apocalypse of Isabella of France », The Art Bulletin. An Illustrated Quarterly, vol. 72, n 2, 1990, p. 224-260.
} 
tourmentés selon le métier qu'ils exercent. Cet ensemble iconographique s'achève à la page suivante (fol. $87 \mathrm{v}$ ), avec une miniature d'une demipage.

Plus bas, dans la même page, se trouve le portrait d'un franciscain, sur le point d'écrire le texte placé sur la droite (fol. 87vb), qui est un commentaire sur l'Apocalypse, également rédigé en ancien français. Son incipit est le suivant : «Ciz Apocalypsis, ce est ceste revelations, il est Jhesu Christi. En ceste parole, ce est Apocalypsis Jhesu Christi, poons nos avoir deus sens : u ke Christe la donet, u k'ele soit donee a Christo ». Le commentaire est dépourvu de miniatures et occupe toute la seconde moitié du volume, jusqu'au fol. 166r.

Ce commentaire français est directement traduit d'un commentaire en latin, qui est issu de l'école d'Anselme de Laon ${ }^{14}$ et transmis par une demi-douzaine de manuscrits ${ }^{15}$. Le texte latin correspond mot pour mot à celui de l'Apocalypse d'Isabelle, comme on peut le constater avec l'exemple suivant :

\section{Commentaire latin ${ }^{16}$}

Hec Apokalipsis, id est revelatio, est Jhesu Christi. In hoc verbo, Apocalipsi Jhesu Christi, duos sensus habere possumus: vel

\section{Traduction $^{17}$}

Ciz Apocalypsis, ce est ceste revelations, il est Jhesu Christi. En ceste parole, ce est Apocalypsis Jhesu Christi, poons nos

\footnotetext{
${ }^{14}$ Cette école du nord de la France a renouvelé l'interprétation de l'Apocalypse dans les années 1090-1120. Voir Guy Lobrichon, «L'Apocalypse en débat : entre séculiers et moines au XII ${ }^{\mathrm{e}}$ siècle (v. 1080-v. 1180) », dans L'Apocalisse nel Medioevo. Atti del Convegno internazionale dell'Università degli Studi di Milano e della Società Internazionale per lo Studio del Medioevo Latino (Gargnano sul Garda, 18-20 maggio 2009), dir. Rossana E. Guglielmetti, Firenze, Edizioni del Galluzzo, 2011, p. 403-426.

${ }^{15}$ Voir Friedrich Stegmüller, Repertorium biblicum Medii Aevi, Madrid, Consejo superior de investigaciones científicas, vol. 2, 1950, p. 117, $\mathrm{n}^{\mathrm{o}} 1371$. Ce commentaire est nettement différent de celui qu'on attribue à Bérengaud, qui lui est contemporain et qui existe dans de nombreuses versions.

${ }^{16}$ Leipzig, Universitätsbibliothek, ms. 173, fol. 1v-2r. Il s'agit de l'un des cinq manuscrits mentionnés. $C f$. ibid.

${ }^{17}$ Paris, BnF, fr. 13096, fol. 87vb.
} 
quod Christus det illam, vel quod Christo data sit. avoir deus sens: $\mathrm{u}$ ke Christe la donet, u k'ele soit donee a Christo.

La parenté entre ces deux textes est indubitable : le texte français est bien une traduction littérale du commentaire latin. Ce dernier comporte également un préambule, qui correspond exactement au prologue français distingué plus haut et situé au début du manuscrit :

\section{Commentaire latin}

Johannes, ob evangelii predicationem tentus a

Domiciano Cesare, ante portam latinam in ferventis olei dolium missus ${ }^{18}$.
Traduction

Johans por le preechement del evangelie fut tenus de Domicien et devant la porte latine mis en un tonel de builhant oile $\mathrm{e}^{19}$.

Le commentaire de l'école d'Anselme de Laon a ainsi été scindé en deux afin que ses premières lignes puissent servir de prologue à une nouvelle traduction de l'Apocalypse (la version A). On peut supposer que la traduction du texte biblique a été effectuée spécialement pour le volume, mais il est possible aussi qu'il s'agisse d'une traduction antérieure, qui aurait simplement été reprise.

\section{L'Apocalypse d'Isabelle de France et son usage}

Le colophon ${ }^{20}$ livre de précieuses informations concernant l'usage de cette Apocalypse. Il se trouve à la dernière page (fol. 167r) et indique que le manuscrit a été exécuté et enluminé par un dénommé "Colin Chadewe» qui l'aurait achevé en l'an 1313, «le samedi après la saint Denis », soit le 13 octobre. Le colophon ne donne le nom d'aucun destinataire. Mais selon l'historienne de l'art Suzanne Lewis ${ }^{21}$, le manuscrit aurait été confectionné par des artistes du nord-est de la France,

\footnotetext{
${ }^{18}$ Leipzig, Universitätsbibliothek, ms. 173, fol. 1v.

${ }^{19}$ Paris, BnF, fr. 13096, fol. 2ra.

${ }^{20}$ Voir supra, note 11.

${ }^{21}$ Suzanne Lewis, « The Apocalypse of Isabella of France », art. cit., p. 224-234.
} 
pour Isabelle de France (1295-1358), la fille du roi de France Philippe IV le Bel (1268-1314). En 1308, Isabelle avait épousé le roi d'Angleterre Édouard II (1284-1327). À sa mort, en 1358, l'inventaire de ses biens mentionnait une Apocalypse enluminée ${ }^{22}$ qui correspondrait, d'après Suzanne Lewis, au manuscrit de la BnF.

Plusieurs indices permettent aussi de penser que le manuscrit a été conçu pour commémorer la visite d'Isabelle et d'Édouard II à Paris en mai-juin 1313. Philippe le Bel organise de grandes festivités à la Pentecôte pour l'adoubement de ses trois fils et doit pour l'occasion confirmer l'engagement qu'il a pris l'année précédente, au Concile de Vienne, de partir en croisade. Parmi les invités se trouvent le roi Édouard II et la reine Isabelle, qui vient de donner naissance au futur Édouard III quelques mois plus tôt, le 13 novembre $1312^{23}$.

Les célébrations organisées par Philippe le Bel à la Pentecôte 1313 ont été relatées par Geoffroi de Paris dans sa Chronique métrique:

Et si vous puis bien creanter

Qu'enfer i fu noir et puant :

Les ames getant et ruant, Dyables i ot plus de cent, Qui tuit sailloient adjecent Por les ames a elz atrere, A cui faisoient maint contraire.

La les creüt on tormenter

Et les veoit on dementer ${ }^{24}$.

Ces vers décrivent une pantomime qui eut lieu lors de ces festivités. On peut ainsi imaginer que les miniatures infernales placées entre l'Apocalypse et son commentaire (fol. 86r-87v) en gardent le

\footnotetext{
${ }^{22}$ Londres, Public Record Office, 31 Edw. III, E101/393/4, fol. 9v.

${ }^{23}$ Il n'est nul besoin de rappeler que les prétentions d'Édouard III au trône de France, en tant que petit-fils de Philippe le Bel, sont l'une des causes de la guerre de Cent Ans.

${ }^{24}$ Geoffroi de Paris, La Chronique métrique attribuée à Geffroy de Paris, éd. Armel Diverrès, Paris, Faculté des lettres de l'université de Strasbourg, 1956, v. 4964-4972 (Paris, BnF, fr. 146, fol. 78vc).
} 
souvenir: des masques sont d'ailleurs suspendus aux quatre angles du frontispice, indiquant l'origine théâtrale de ces enluminures. Si le manuscrit était bien destiné à Isabelle de France, il n'est pas anodin non plus qu'une femme figure au cœur du second frontispice (fol. 86v), pour illustrer le péché de vanité.

Ainsi, la copie de l'Apocalypse d'Isabelle de France aurait commémoré la visite d'Isabelle à Paris en mai-juin 1313. Mais au-delà de cette dimension personnelle, l'ouvrage possède également une portée politique. Au fol. 50r par exemple, figure une enluminure qui représente Apocalypse, XVI, 12-14. Ces versets évoquent la lutte des rois contre la Bête, le Dragon et le Faux Prophète. En haut à droite de l'enluminure, on peut reconnaître le roi de France à son blason, accompagné du roi d'Angleterre. La présence de ces deux souverains permet d'insister sur le lien dynastique entre la France et l'Angleterre. Plus bas dans l'enluminure, sur la gauche, l'empereur des Romains est également présent. L'enluminure ferait donc écho à la promesse de croisade de Philippe le Bel, confirmant la portée politique conférée au texte biblique $^{25}$.

\section{Transmission de la version A dans les Bibles historiales}

En introduction, nous indiquions que la version A de l'Apocalypse d'Isabelle de France se trouvait aussi dans un ensemble de six Bibles historiales. Paul Meyer ne connaissait que trois d'entre elles en 1901 :

- Londres, BL, Royal 17.E.VII, vol. 2, fol. 235r-241r ;

- Paris, BnF, fr. 2, fol. 505r-511v ;

- Paris, BnF, fr. 5707, fol. 358r-367v.

Il convient d'ajouter les trois manuscrits suivants :

\footnotetext{
${ }^{25}$ Pour plus de précisions concernant la portée politique de l'ouvrage, voir Suzanne Lewis, «The Apocalypse of Isabella of France », art. cit., p. 226-230.
} 
- Berlin, Staatsbibliothek, Cod. Philipps 1906, fol. 498r-501v ${ }^{26}$;

- Eton, College Library, ms. 3, fol. 235v-241v;

- La Haye, Musée Meermann Westreenen, ms. 10 B 23, fol. 573v-580r.

Le contexte manuscrit n'est plus le même que celui de l'Apocalypse d'Isabelle de France. Dans les Bibles historiales, l'Apocalypse n'est pas autonome, comme c'est le cas dans le manuscrit d'Isabelle de France. Le texte est placé à la fin du manuscrit, après les autres livres de la Bible, et suit donc le reste de la Bible sans solution de continuité.

La date de réalisation apparaît dans quatre de ces Bibles $^{27}$, permettant d'affirmer que la version $\mathrm{A}$ a été utilisée sur une période relativement brève, entre 1357 et 1371. Cette datation correspond globalement au moment où Charles V est à la tête du royaume de France. Certaines de ces Bibles ont d'ailleurs été expressément effectuées pour Charles V, puisqu'il figure comme dédicataire sur plusieurs miniatures ${ }^{28}$.

Si l'on met de côté le manuscrit de Berlin, où l'Apocalypse comporte une vingtaine de miniatures, celle des cinq autres Bibles historiales n'est pas pourvue d'un programme iconographique, à la différence du manuscrit fr. 13096. L'Apocalypse y est simplement

\footnotetext{
${ }^{26}$ Le début de l'Apocalypse manque dans le manuscrit de Berlin : le texte commence par Apocalypse, VI, 2. Nous reviendrons plus loin sur ce manuscrit.

${ }^{27}$ Le manuscrit Royal 17.E.VII se termine sur un acrostiche vertigineux, qui révèle sa date de confection quand on lit la première lettre de chaque vers du bas vers le haut : «MILE/TROIS/CENS/CINQUANTE/SEPT » (vol. 2, fol. 241rc). Le manuscrit fr. 5707, qui a sûrement été copié sur le manuscrit de Londres, précise que l'ouvrage «fu parfait, que je ne mente/L'an mil .ccc. trois et .lx.» (fol. 368rb). La Bible historiale de Berlin «fu parfaite en l'a[n] mccclxviii » (fol. 501). La Bible historiale de La Haye a, quant à elle, été confectionnée «anno domini millesimo trecentesimo septuagesimo primo » (fol. 1v).

${ }^{28}$ Le manuscrit de La Haye, par exemple, a été réalisé en 1371 pour Charles V, alors roi de France: au fol. 1v, on peut lire que le manuscrit a été confectionné «ad preceptum ac honorem illustri principis karoli regis francie ». Voir aussi le portrait de Charles V qui figure dans le manuscrit fr. 5707, fol. 368ra. Sur ces deux manuscrits, voir Léopold Delisle, Recherches sur la librairie de Charles V, Paris, Champion, 1907 , p. $148-149$ et $153-156$.
} 
surmontée d'une miniature introductive, comme c'est le cas pour tous les autres livres de la Bible. Cette miniature, qui représente saint Jean et le Dragon à sept têtes, est reproduite à l'identique dans chaque Bible historiale.

\section{Comparaison textuelle entre $\mathbf{A}$ et $\mathbf{A}^{\prime}$}

Après avoir indiqué le contexte historique et iconographique de ces Apocalypses, il faut désormais se pencher sur le texte qu'elles donnent. Comme nous l'avons dit plus haut, l'Apocalypse d'Isabelle de France et ce groupe de six Bibles historiales contiennent la même traduction. Toutefois, on constate quelques divergences textuelles qui permettent de préciser la filiation entre la version A (l'Apocalypse de 1313) et une version qui en dérive et que nous appellerons $\mathrm{A}^{\prime}$ (Bibles historiales).

Dans l'ensemble, le texte que donne l'Apocalypse d'Isabelle (A) est de meilleure qualité que celui des Bibles historiales (A'), comme le prouve l'exemple suivant :

Vulgate erat numerus eorum millia millium ${ }^{29}$

A leur nonbres astoit milh milhier ${ }^{30}$

$\mathbf{A}^{\prime} \quad$ leur nombres estoit trois milliers ${ }^{31}$

Les deux textes n'indiquent pas le même nombre d'anges («milh» vs. «trois »), mais c'est l'Apocalypse d'Isabelle qui comporte la bonne leçon («millia millium»). Cette divergence est probablement due à l'erreur d'un copiste, qui aura confondu «.m. » et «.iii. ». Cela suppose qu'il y ait eu une copie intermédiaire entre $\mathrm{A}$ et $\mathrm{A}^{\prime}$, dans laquelle les nombres étaient indiqués en chiffres romains.

\footnotetext{
${ }^{29}$ Apocalypse, $v, 11$. Nous citons le texte biblique d'après la Vulgate SixtoClémentine: Biblia sacra iuxta vulgatam clementinam. Nova editio [1946], éd. Alberto Colunga et Lorenzo Turrado, Madrid, Biblioteca de autores cristianos, 1999.

${ }^{30}$ Paris, BnF, fr. 13096, fol. 14va.

${ }^{31}$ Paris, BnF, fr. 5707, fol. 360ra.
} 
L'exemple suivant vient confirmer cette hypothèse :

\section{Vulgate bilibris tritici denario et tres bilibres hordei denario $^{32}$}

A une bilivre de froment pour un donier

et trois bilivre d'orge por un donier ${ }^{33}$

$\mathbf{A}^{\prime} \quad$ deux livres de fourment pour un denier

et trois livres d'orge pour un denier ${ }^{34}$

La version A donne une traduction littérale, calquée sur le latin : bilibris (adjectif qui désigne un poids égal à deux livres) est traduit par «bilivre». Dans la version $\mathrm{A}^{\prime}$ en revanche, le terme «bilivre» est remplacé par «deux livres». Il y aurait donc eu, de $\mathrm{A}$ à $\mathrm{A}^{\prime}$, un phénomène de révision censé rendre le texte de l'Apocalypse plus facile d'accès. On pourra toutefois s'étonner de voir que les «trois bilivre » d'orge de la version A ne sont pas devenues «six livres » dans la version $\mathrm{A}^{\prime}$, mais « trois livres ». Cette erreur, que l'on rencontre dès le manuscrit fr. 5707 de la BnF, s'explique là encore d'un point de vue paléographique : «trois » transcrit le chiffre «.iij. », qui lui-même est peut-être la corruption d'un «.uj.» antérieur (pour six). De «.uj. » à «.iij. », il n'y a qu'un pas, ou, devrions-nous dire, il n'y a qu'un empattement ${ }^{35}$. Une fois de plus, nous devons en induire qu'il existait une copie intermédiaire de cette traduction, permettant aux ateliers parisiens, au milieu des années 1350, de travailler sur le texte d'Isabelle de France.

\footnotetext{
${ }^{32}$ Apocalypse, vi, 6.

${ }^{33}$ Paris, BnF, fr. 13096, fol. 15vb.

${ }^{34}$ Paris, BnF, fr. 5707, fol. 360rb.

${ }^{35}$ Il est facile de confondre « $u$ » et « $11 »$, du fait de l'empattement dont est pourvue la lettre « $1 »$ dans l'écriture gothique.
} 


\section{La version A, substitut à la version CG ?}

S'il importe de savoir comment la traduction biblique du manuscrit fr. 13096 a pu se retrouver dans plusieurs Bibles historiales, il est capital aussi de comprendre pourquoi.

Pourquoi rencontre-t-on la version A dans un groupe de six Bibles historiales à la fois proches dans le temps et dans l'espace? Cette question mérite d'être soulevée dans la mesure où la traduction que les Bibles historiales renferment n'est généralement pas la version $\mathrm{A}$, mais la version $\mathrm{C}$, pour laquelle nous avons au moins 63 témoins. Ce succès s'explique par le fait qu'elle a été réalisée dans le cadre de la Bible du XIII siècle $^{36}$, dont le second volume a été utilisé au début du XIV ${ }^{\mathrm{e}}$ siècle pour compléter la Bible historiale (1294) de Guyart des Moulins ${ }^{37}$. En raison de cette filiation, les Bibles historiales se terminent presque systématiquement sur le texte de la version $\mathrm{C}$.

Cependant, à une date indéterminée, peut-être vers 1310-1315, une version hybride est apparue, que Paul Meyer appelle composite ${ }^{38}$ et que nous avons conservée dans 22 manuscrits. Le caractère hybride de cette version, qu'Antoine Vérard imprime par mégarde vers 1498-1499 ${ }^{39}$,

\footnotetext{
${ }^{36}$ Sur la Bible $d u$ XIII ${ }^{e}$ siècle, on consultera en priorité les travaux de Clive R. Sneddon, notamment l'article suivant, où sont recensées les principales sources bibliographiques sur le sujet: Clive R. Sneddon, «The Old French Bible : the first complete Vernacular Bible in Western Europe », dans The Practice of the Bible in the Middle Ages. Production, reception, and performance in Western Christianity, dir. Susan Boynton et Diane J. Reilly, New York, Columbia University Press, 2011, p. 296-314.

${ }^{37}$ Sur la Bible historiale, on lira avec intérêt les travaux d'Akiko Komada, d'Éléonore Fournié et de Xavier-Laurent Salvador. Pour une synthèse récente sur le sujet, voir l'article de Guy Lobrichon, "The Story of a Success. The Bible historiale in French (1295-ca. 1500) », dans Form and Function in the Late Medieval Bible, dir. Eyal Poleg et Laura Light, Leiden/Boston, Brill, 2013, p. 307-331.

${ }^{38}$ Voir Léopold Delisle et Paul Meyer, L'Apocalypse en français au XIII siècle, op. cit., p. CCXLVI-CCXLVII.

${ }^{39}$ Cet incunable est décrit par Éléonore Fournié dans «Catalogues des éditions de la Bible historiale », L'Atelier du Centre de recherches historiques, 3/2, 2009, $\mathrm{n}^{\mathrm{o}} 1$ : https://acrh.revues.org/1839\#tocto1n1.
} 
s'explique par le fait qu'elle combine les versions $C$ et $G$ (aussi l'appellerons-nous plus simplement CG) : elle donne d'abord le texte de C jusqu'à Apocalypse, XI, 12, puis le texte de G à partir d'Apocalypse, XI, 14. La première moitié du texte n'est donc pas glosée, contrairement à la seconde, comme le montre le schéma suivant :

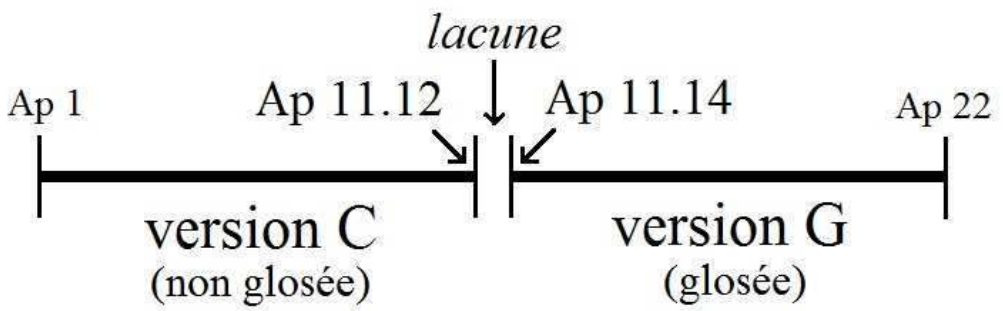

À l'origine de cet accident se trouve probablement une Petite Bible historiale complétée dont l'Apocalypse est lacunaire ou fautive ${ }^{40}$. Un copiste a sûrement complété le texte à partir d'un autre manuscrit, qui contenait en réalité la version $\mathrm{G}^{41}$. Et il ne semble pas s'être soucié du fait que cette version était glosée. Même Antoine Vérard n'éprouve aucun scrupule à imprimer cette version à moitié glosée. Toutefois, on peut imaginer que dans les années 1350 , les ateliers qui travaillent pour le futur Charles $\mathrm{V}$ sont réticents à copier un texte qui est manifestement

\footnotetext{
${ }^{40}$ Les Bibles historiales contenant une Apocalypse fautive sont nombreuses. Dans une dizaine de manuscrits par exemple, la version $\mathrm{C}$ de l'Apocalypse apparaît par blocs désordonnés : Apocalypse, I, 1-II, 17 ; VI, 17-XI, 4 ; II, 17-VI, 17 ; XIV, 14-XVIII, 13 ; XI, 5-XIV, 14; XVIII, 13-XXII, 21. Les sauts interviennent au milieu de la page, laissant supposer que ce désordre a été causé par un défaut de reliure à un stade antérieur de la transmission. Le ms. 5059 de la Bibliothèque de l'Arsenal présente un phénomène similaire : il s'agit d'une Petite Bible historiale complétée, datée de 1317, dans laquelle le texte est donné dans l'ordre suivant: Apocalypse, I, 1-IX, 20 ; XII, 14-XIV, 20 ; IX, 20-XII, 14 ; XVII, 16-XIX, 18 ; XV, 1-XVII, 16 ; XIX, 19-XXII, 21. La version composite se justifierait donc par la volonté de remédier à une discontinuité ou à une lacune dans un manuscrit source.

${ }^{41}$ D'ailleurs, au sein d'un même manuscrit, il n'est pas rare de voir coexister deux versions différentes : c'est le cas par exemple du manuscrit n. a. f. 6883 de la BnF, qui donne successivement les versions G (fol. 88ra-131ra) et C (fol. 132ra-146rb).
} 
fautif. On peut donc supposer qu'ils ont eu l'intention de remplacer la version $\mathrm{CG}$ par une version de meilleure qualité, à savoir la version $\mathrm{A}$.

De ce point de vue, l'une des six Bibles historiales que nous avons mentionnées (Berlin, Staatsbibliothek, Cod. Philipps 1906, fol. 498r501v) livre un indice décisif. Nous n'avons pas pu consulter ce manuscrit, mais selon la notice qu'en donne Clive R. Sneddon ${ }^{42}$, le texte juxtapose deux versions différentes : la version CG jusqu'à la fin d'Apocalypse, XII, puis la version A à partir d'Apocalypse, XIII. Le manuscrit réunit ainsi trois versions : la version C (non glosée) jusqu'à Apocalypse, XI, 12, la version G (glosée) pour la fin du chapitre XI et le chapitre XII, la version A (non glosée) à partir d'Apocalypse, XIII. Le scribe aurait commencé à copier une Bible historiale dont l'Apocalypse était composite, mais en copiant la fin du chapitre XI et le chapitre XII, il aurait pris conscience du fait que son modèle était fautif, le texte devenant brusquement glosé ; afin d'y remédier, le copiste aurait tout à fait pu vouloir remplacer la version composite par une autre de meilleure qualité, la version A, comme l'a fait son devancier. Deux copistes attentifs du milieu du XIV ${ }^{\mathrm{e}}$ siècle auraient donc eu la volonté, indépendamment l'un de l'autre, d'amender le texte de la Bible historiale en ayant recours à une version appréciée pour son apparente fidélité, à savoir la version A. Ces indices confirmeraient ainsi notre hypothèse selon laquelle l'Apocalypse d'Isabelle de France circule à cette époque par l'intermédiaire de copies.

Le manuscrit Paris, BnF, fr. 13096 a donc connu une destinée singulière, puisqu'il a probablement été réalisé à Paris en 1313, en guise de cadeau de la part de Philippe IV à sa fille, Isabelle de France, devenue reine d'Angleterre. La traduction que ce manuscrit contient semble avoir

\footnotetext{
${ }^{42}$ Clive R. Sneddon, A critical edition of the four Gospels in the Thirteenth-Century Old French translation of the Bible, thèse de l'université d'Oxford, 1978, 2 vol., vol. 1, p. 291.
} 
été utilisée, au milieu du XIV ${ }^{\mathrm{e}}$ siècle, pour remplacer une version fautive de l'Apocalypse au sein de la Bible historiale. Cette substitution a eu lieu à un moment déterminant de l'histoire du royaume de France puisqu'en 1357, date de la première Bible historiale pourvue d'une version A (Paris, $\mathrm{BnF}$, fr. 5707), le futur Charles $\mathrm{V}$ accède au pouvoir, à la suite de l'emprisonnement du roi de France Jean II par les Anglais.

Bien qu'il s'agisse sûrement d'une coïncidence chronologique, il n'en demeure pas moins que la transmission de la version A soulève des interrogations qu'il serait difficile de résoudre avec certitude. L'Apocalypse d'Isabelle de France a-t-elle fait l'objet de copies en Angleterre, qui ultérieurement auraient été «rapatriées» sur le continent? L'ouvrage a-t-il été copié une seconde fois en France avant d'être offert au couple royal d'Angleterre ${ }^{43}$ ? On pourrait même se demander, en dépit des nombreux indices qui plaident en faveur de ce postulat, si le manuscrit en question était bien destiné à Isabelle: le manuscrit fr. 13096 de la BnF correspond-il vraiment à l'Apocalypse mentionnée par l'inventaire d'Isabelle en 1358? La date de 1357, que l'on trouve dans le manuscrit fr. 5707 de la $\mathrm{BnF}$, serait un indice susceptible d'infirmer cette hypothèse, malgré la probabilité de copies intermédiaires entre les manuscrits fr. 13096 et fr. 5707.

Quoi qu'il en soit, dans l'éventualité où le manuscrit fr. 13096 ait véritablement appartenu à la reine d'Angleterre, force est de constater que la concorde franco-anglaise affichée dans l'une des miniatures du manuscrit (fol.50r, voir supra) n'a plus lieu d'être en 1357, vingt ans après le début de la guerre de Cent Ans. Le fait de retrouver la traduction qui aujourd'hui porte son nom dans des Bibles historiales auréolées par la

\footnotetext{
${ }^{43}$ Le manuscrit ayant été achevé en octobre 1313, Isabelle de France, venue quelques mois plus tôt à Paris, était déjà retournée en Angleterre : l'ouvrage n'a pu donc être offert qu'à une date plus tardive.
} 
présence de Charles V nous rappelle que la lutte entre la France et l'Angleterre s'exerce aussi à l'échelle des lettres, et que la transmission du texte biblique est intimement liée à la question du pouvoir politique. La forme des Bibles est toujours tributaire de l'usage qui en est fait. 
